\title{
Size Effect on Strength of Micron-sized Polycrystals - a Dislocation Dynamics Simulation Study
}

\author{
P.S.S. Leung ${ }^{\S}$ and A.H.W. Ngan \\ Department of Mechanical Engineering, \\ The University of Hong Kong, Pokfulam Road, Hong Kong, P.R. China \\ ${ }^{\S}$ Corresponding author (email: peggylss927@gmail.com)
}

\begin{abstract}
The effects of grain size $(d)$, specimen size $(t)$ and initial dislocation density on the strength of micron-sized polycrystals are simulated by $2 \mathrm{D}$ dislocation dynamics. The results show that the continuum phenomenon of "smaller being weaker" effect for $1<t / d \widetilde{<} 3$ occurs only when the initial dislocation density is high. When $t / d<1$ with high initial dislocation density, or when the initial dislocation density is low regardless of $t / d$, the opposite "smaller being stronger" effect occurs.
\end{abstract}

Keywords: polycrystalline; simulation; dislocations 
It is well-known that the strength of bulk-sized polycrystalline metals depends on the grain size according to the Hall-Petch relation [1,2], and when the grain size falls in the nanometric range, the inverse Hall-Petch effect may occur [3]. On the other hand, whereas the strength of single crystalline specimens of micron sizes in general varies as the negative power of the specimen size [4-15], the effect of specimen size on strength for polycrystalline specimens has not been well understood. The domain of special interest is when there are only a few grains across the specimen thickness, so that the mean-field condition as in the Hall-Petch or inverse Hall-Petch effect breaks down. In this case, the grain size $(d)$ and specimen size $(t)$ can be expected to produce interesting coupled effects to control strength.

The available experimental results in the above-mentioned regime of $t / d \sim 2$ to 4 have been controversial. In one group of observations, a "smaller being weaker" effect was reported, where strength decreases as the specimen size $(t)$ decreases. Keller and Hug [16] reported that for polycrystalline nickel thin films of millimeter thickness, the flow stress of specimens with $t / d<$ 3.6 becomes significantly smaller than the Hall-Petch trend extrapolated from specimens with $t / d>3.6$ at 0.14 strain in the work-hardening regime. In another report on the same material system [17], the same group of authors found that at a fixed grain size of either $100 \mu \mathrm{m}$ or $500 \mu \mathrm{m}$, decreasing the $t / d$ ratio from 4 to 1 decreases the tensile strength [17], which agrees with the results of Miyazaki et al. [18] and Wang et al. [19]. Some other researchers reported the opposite trend of "smaller being stronger". Chen and Ngan [20] reported that for silver wires of diameters ranging from 20 to $50 \mu \mathrm{m}$, the "smaller being stronger" effect was observed for $t / d<3$. Chauhan and Bastawros [21] studied size effect of strength using freestanding copper thin films, and discovered that for grain size below $5 \mu \mathrm{m}$, the "smaller being stronger" effect would be exhibited. Mahabunphachai and Koç [22] pointed out, from the literature, that there is a transition from the "smaller being weaker" to the "smaller being stronger" trend at $t / d \sim 2$ to 4 . Raulea et al. [23] reported a consistent "smaller being weaker" trend for $t / d>1$, but when $t / d<1$, the yield strength would increase with increasing grain size. For these different size effects on strength reported by different researchers, Janssen, Geers and co-workers [24, 25] suggested a classification scheme into microstructural or intrinsic size effects, statistical size effects, lattice curvature and strain gradients, surface or interfacial constraints and geometrical effects, with possible competition between them. Also, in Fig. 41 of the review paper by Greer and De Hosson [14], the combined 
effects of extrinsic and intrinsic length scales on strength of nanocrystalline Ni are presented, which clearly show both trends of smaller being weaker and stronger, depending on the grain size.

In this study, 2D dislocation dynamics simulation, a common tool for studying the dependence of crystalline deformation on microstructural factors [26-30], was employed to study the effects of grain size $(d)$, specimen size $(t)$, initial dislocation density and initial intragranular dipole source density on the strength of micron-sized polycrystalline structures. In these simulations, edge dislocations are randomly placed in a rectangular simulation region with a given initial density. Grains are rectangular domains partitioning the simulation region, and grain boundaries are set to be impenetrable by dislocations. Each grain contains 2 slip systems oriented at $\phi$ and $\phi+60^{\circ}$ measured from the horizontal reference $x$-axis, where $\phi$ is randomly assigned. Tensile load is applied along the vertical direction, along which periodic boundary conditions are used for the stress field. The left and right sides of the simulation region are free surfaces with image stresses applied. The free surface is meshed into rectangular strips, and external line forces are applied to counteract the dislocation forces on the surface, in analogy to how surface tractions are removed in 3D calculations [31]. The stress field around the line forces would be the image stress acting on the dislocations [32]. Benzerga [33] proposed a ' $2.5 \mathrm{D}$ ' method to consider the contribution of forest dislocation interactions in 2D DD simulations. Here, in a similar spirit, a friction stress $\tau_{\text {forest }}^{i}$ equivalent to the bow-out shear stress in forest interaction is applied to resist the travelling dislocations according to

$$
\tau_{\text {forest }}^{i}=\frac{\alpha \mu b}{l^{i}}
$$

where $l^{i}$ is the average spacing of dislocations in grain $i$, given by the dislocation density $\rho_{\text {mobile }}^{i}$ in the grain as

$$
l^{i}=\frac{1}{\sqrt{\rho_{\text {mobile }}^{i}}} .
$$

Here, the forest dislocations are assumed to be the mobile population for simplicity, $\mu$ is shear modulus, and $b$ the Burgers vector. Dislocation velocities are calculated via 


$$
v=v_{0}\left(\frac{\tau}{\tau_{0}}\right)^{m}
$$

where $v_{0}, \tau_{0}$ and $m$ are constants, and $\tau$ is the net stress acting on the slip system of the dislocation, which is the sum of the applied stress, interaction stresses from other dislocations, resistance to overcome forest interactions $\tau_{\text {forest }}^{i}$, and image stresses from free surfaces. The Peierls-Nabarro friction stress is ignored in this investigation. Two dislocations of opposite signs would annihilate at a cutoff distance of $8 b$. Dislocations also annihilate at free surfaces. In terms of dislocation generation, both grain-boundary (GB) and intragranular dipole sources are used. The GB source density is expressed as the number of sources per unit length of GB, and the intragranular source density is the number of sources per unit area. Each grain is divided into a GB region, defined as the area within $250 b$ to the grain boundary, and the remaining intragranular region, as illustrated in Figure 1. The two types of sources are placed randomly in these two regions respectively. Whenever the resultant stress acting on a dipole source exceeds its nucleation stress $\tau_{n u c}$ for a continuous period of 10 time steps, a dislocation dipole will be nucleated at the same site and on the same slip plane of the source. The material properties of silver are used, where the shear modulus is $30 \mathrm{GPa}$ and the Poisson ratio is 0.37 . The nucleation stress $\tau_{n u c}$ here follows a Gaussian distribution with mean of mean $0.006 \mu / 2 \pi$ (around $30 \mathrm{MPa}$ ) and standard deviation $0.0005 \mu / 2 \pi$ (around 2.5MPa), close to the values of Nicola et al. [27]. To ensure that the two members of the nucleated dipole do not annihilate immediately, they are spaced $L_{n u c}$ apart, where $L_{n u c}=\frac{1}{2 \pi(1-v)} \frac{\mu b}{\tau_{n u c}}$, so that the nucleation stress would just balance the attraction between them. After each nucleation, a recovery time of 10 time steps is required before the next nucleation.

The model parameters used in the simulations are normalized following an earlier scheme [34]. First, stress $\tau$ is normalized by $\mu / 2 \pi(1-v)$, where $\mu$ is the shear modulus, i.e.

$$
\tilde{\tau}=\frac{\tau}{\frac{\mu}{2 \pi(1-v)}}
$$

Distances and lengths $x$ are normalized by the Burgers vector $b$, i.e.

$$
\tilde{x}=\frac{x}{b} .
$$


The dislocation velocity $v$ is assumed to obey the law in Eqn. (3), and in the simulation programme, the normalized velocity used is

$$
\tilde{v}=\frac{\tilde{\tau}}{\tilde{\tau}_{0}}
$$

where $\tilde{\tau}_{0}$ is the normalized stress which would give a normalized velocity equal to $1 \mathrm{~b} /$ time step. In real FCC metals, for example, the exponent $m$ in Eqn. (3) is very large, but previous DD simulations usually employed a value of unity for $m$ for the sake of simplicity, and so for this reason, $m$ is also set to be 1 in the present work. $\tilde{\tau}_{0}$ is set to be $10^{-4}(1-v)$. From Eqns. (3) and (6), the normalized velocity is related to the real velocity by:

$$
\tilde{v}=\frac{v}{v_{0}} \cdot\left(\frac{2 \pi(1-v) \tau_{0}}{\mu}\right) \cdot \frac{1}{\tilde{\tau}_{0}}
$$

The normalization of distance and velocity according to Eqns. (5) and (7) respectively also uniquely specifies the way time $t$ is normalized:

$$
\widetilde{\Delta t}=\frac{v_{0}}{b} \cdot \frac{\mu}{2 \pi(1-v) \tau_{0}} \cdot \tilde{\tau}_{0} \cdot \Delta t
$$

A high and a low dislocation density scenario were simulated as follows:

(i) HIGH density case - both the initial dislocation density and intragranular dipole source density were set to be $2.5 \times 10^{-6} b^{-2}\left(\sim 10^{13} \mathrm{~m}^{-2}\right)$;

(ii) LOW density case - both the initial dislocation and dipole source density were $6.25 \times 10^{-8} b^{-2}\left(\sim 10^{12} \mathrm{~m}^{-2}\right)$.

The grain boundary dipole source density is $0.00025 b^{-1}$ for both cases. For all the simulations in this study, the lengths of the specimens were constant at $72000 \mathrm{~b}$. The width was varied to study the effect of thickness $(t)$. For each combination of grain size, thickness and initial densities, five simulations with different randomly generated grain orientations and initial dislocations at the same density were performed. The yield stress data presented below is the average of the five simulations.

Figure 2 shows typical stress-strain curves when the grain size is kept constant at $3000 \mathrm{~b}$ while the thickness varies. At a high density setting (Figure 2a), the "smaller being weaker" trend is consistently followed until the $t / d$ ratio drops below 1. At low densities (Figure 2b), the 
"smaller being stronger" trend is exhibited for the whole range of $t / d$ ratios simulated, although the trend is more obvious for small $t / d$ ratios. Figure 3 a shows plots of the $0.2 \%$ proof stress $\sigma_{y}$ vs $d^{-1 / 2}$ for the high density case. For all grain sizes, as long as $t / d>1$, a "smaller being weaker" trend is observed. However, when the $t / d$ ratio drops below 1, corresponding to a bamboo arrangement of the grains, a consistent and significant "smaller being stronger" effect is observed. The switch between the two opposite size effects as the $t / d$ ratio drops below 1 is associated with a reduction of the Hall-Petch slope. Note that for $12000 b \leq t \leq 36000 b$, the 3 curves have larger and almost constant Hall-Petch slope, while for $3000 b \leq t \leq 6000 b$, the curves become bilinear with a smaller slope in the $t / d<1$ regime, and the $t=1500 \mathrm{~b}$ curve has a smaller and almost constant slope similar to that of the $3000 b \leq t \leq 6000 b$ in the $t / d<1$ regime. Figure $3 \mathrm{~b}$ shows the $\sigma_{y}$ vs $d^{-1 / 2}$ plots for the low initial dislocation density case. Unlike the high initial density case in Figure 3a, the "smaller being stronger" trend is observed for all values of $t / d$ ratio simulated, even when it is $>1$. Also, the Hall-Petch relation is in general not obeyed with nonlinear $\sigma_{y}$ vs $d^{-1 / 2}$ trends, as well as the occurrence of inverse Hall-Petch effect as indicated in Figure 3b.

Figure $4 \mathrm{a}$ and $4 \mathrm{~b}$ shows the stress and residual dislocation distribution for the high initial dislocation density scenario. When $t / d>1$ (Figure 4a), the surface grains are under lower stresses than the interior grains, because surface grains have less dislocation pile-ups and hence less stress concentration. When $t / d \leq 1$ (Figure $4 \mathrm{~b}$ ), the stress distribution is more random within the sample. The absence of the longitudinal (vertical) grain boundaries in the $t / d \leq 1$ case gets rid of a lot of sites for dislocation pile-up, this results in a reduction of the Hall-Petch slope for $t / d \leq 1$ compared with $t / d>1$ as seen from Figure 3a. Also, in Figure $4 \mathrm{~b}$, the dislocation density in the thinner $t=1500 \mathrm{~b}$ specimen is much lower than that in the thicker $t=12000 \mathrm{~b}$ specimen. This indicates that dislocations are continuously disappearing from the thinner specimen during deformation in the $t / d \leq 1$ regime, and the lack of dislocations leads to the "smaller being stronger" effect.

Figure $4 \mathrm{c}$ shows the stress and residual dislocation distribution at $0.2 \%$ strain when the initial dislocation density is low. It can be seen that the grain boundaries become the softening 
region, because plasticity is achieved by the operation of GB dipole sources only. Thus, a smaller specimen has fewer grain boundaries and hence the strength is higher.

The present simulations show that the deformation of polycrystals with a few grains across their thickness may exhibit two distinctive types of behavior. In the first type which is conventional, deformation is strain-hardening dominated. This happens when the initial dislocation density is high and the specimen size is also large (Figure 4a). In this case, the normal Hall-Petch behavior always occurs since the grain boundaries are sites for dislocation pile-ups and stress concentration. The "smaller being weaker" size effect [16-19] also occurs when $1<t / d \widetilde{<} 3$ (the right side of Figure $3 \mathrm{a}$ ), since the surface grains are softer as dislocations can disappear from them easily, and a smaller specimen has a higher volume fraction occupied by surface grains. In real experiments, the strain incompatibilities between soft and hard grains would restrain substantial deformation in softer grains [35,36], which would also lead to a "smaller being weaker" trend. In the second type of behavior, plasticity is source limited, and the size effect is the "smaller being stronger" type. This may happen when the specimen size is small although the initial dislocation density may not be low (left side of Figure 3a), or when the initial dislocation density is low for a wider range of specimen sizes (Figure 3b).

To conclude, the present simulations show that in polycrystals, both "smaller being stronger" and "smaller being weaker" size effects may occur, depending on $t / d$ ratio, the specimen size, as well as the initial dislocation density. The work described in this paper was supported by funding from the Research Grants Council (Project No. 7159/10E).

\section{References}

[1] E.O. Hall , Proc. Phys. Soc. Lond. B64 (1951) 747.

[2] N.J. Petch, J. Iron Steel Inst. 174 (1953) 25.

[3] A.H. Chokshi, A. Rosen, J. Karch and H. Gleiter, Scripta Metall. 23 (1989) 1679-1684.

[4] S.S. Brenner, J. Appl. Phys. 27 (1956) 1484. 
[5] G. Richter, K. Hillerich, D.S. Gianola, R. Monig, O. Kraft, C.A. Volkert, Nano Lett., 2009, 9 (8), 3048-3052.

[6] M.D. Uchic, D.M. Dimiduk, J.N. Florando, W.D. Nix, Science 305 (2004) 986.

[7] J.R. Greer, W.C. Oliver, W.D. Nix, Acta Mater. 53 (2005) 1821.

[8] K.S. Ng, A.H.W. Ngan, Scripta Mater. 59 (2008) 796.

[9] D. Kiener, W. Grosinger, G. Dehm, R.Pippan, Acta Mater. 56 (2008) 580.

[10] J.-Y. Kim, D. Jang, J.R. Greer, Scripta Mater. 61 (2009) 300.

[11] J.-Y. Kim, D. Jang, J.R. Greer, Int. J. Plast. 28 (2012) 46.

[12] J.-Y. Kim, J.R. Greer, Acta Mater. 57 (2009) 5245.

[13] K.S. Ng, A.H.W Ngan, Acta Mater. 56 (2008) 1712.

[14] J.R. Greer, J.Th.M. De Hosson, Prog. Mat. Sci. 56 (2011) 654.

[15] R. Dou, B. Derby, Scripta Mater. 61 (2009) 524.

[16] C. Keller, E. Hug, Mater. Lett. 62 (2008) 1718.

[17] C. Keller. E.hug, X. Feaugas, Int. J. Plast. 27 (2011) 635.

[18] S. Miyazaki, K. Shibata, H. Fujita, Acta Metall. 27 (1979) 855.

[19] C.-J. Wang, B. Guo, D.-B. Shan, L.-N. Sun, Trans. Nonferrous Met. Soc. China 19 (2009) 511.

[20] X.X. Chen, A.H.W Ngan, Scripta Mater. 64 (2011) 717.

[21] S. Chauhan, A.F. Bastawros, Appl. Phys. Lett. 93 (2008) 041901.

[22] S. Mahabunphachai, M. Koç, Int. J. Mach. Tool Manu. 48 (2008) 1014.

[23] L.V. Raulea, L.E. Govaert, F.P.T. Baaijens, Advanced Technology of Plasticity, Proceedings of the 6th ICTP, Vol. 2, 939-944, Sept 19-24, 1999. 
[24] P.J.M. Janssen, Th. H. de Keijser, M.G.D. Geers, Mater. Sci. Eng. A 419 (2006) 238.

[25] M.G.D. Geers, W.A.M. Brekelmans, P.J.M. Janssen, Int. J. Solids Struct. 43 (2006) 7304.

[26] L. Nicola, E. Van der Giessen, A. Needleman, Philos. Mag. 85 (2005) 1507.

[27] L. Nicola, E. Van der Giessen, A. Needleman, J. Appl. Phys. 93 (2003) 5920.

[28] R. Kumar, L. Nicola, E. Van der Giessen, Mater. Sci. Eng. A 527 (2009) 7.

[29] H. Fan, Z. Li, M. Huang, X. Zhang, Int. J. Solids Struct. 48 (2011) 1754.

[30] Z. Li, C. Hou, M. Huang, C. Ouyang, Comp. Mater. Sci. 46 (2009) 1124.

[31] M.C. Fivel, G.R. Canova, Model. Simul. Mater. Sci. Eng. 7 (1999) 753

[32] K.L. Johnson, Contact Mechanics, Cambridge University Press, Cambridge, 1985.

[33] A. A. Benzerga, J. Mech. Phys. Solids 57 (2009) 1459.

[34] K.W. Siu, A.H.W. Ngan, Philos. Mag. 91 (2011) 4367.

[35] O. Wouters, W.P. Vellinga, R. van Tijum, J.Th.M. de Hosson, Acta Mater. 53 (2005) 4043

[36] O. Wouters, W.P. Vellinga, R. van Tijum, J.Th.M. De Hosson, Acta Mater. 54 (2006) 2813 


\section{Figure captions}

Figure 1. Grain boundary region and intragranular region.

Figure 2.Stress strain curves of (a) HIGH dislocation density, (b) LOW dislocation density cases (see text for details), grain size $=3000 \mathrm{~b}$.

Figure 3. $\sigma_{y}=d^{-1 / 2}$ plots for (a) HIGH dislocation density, (b) LOW dislocation density cases. Yield strain $=0.2 \%$. The numbers in parentheses are the $t / d$ ratios.

Figure 4. $\tilde{\sigma}_{y y}$ stress distribution contour map and dislocation structures at $0.2 \%$ strain for (a) grain size $d=3000 b$, thickness $t=12000 b$ and $3000 b$, HIGH initial dislocation density case. Stress is normalized by the value $\tilde{\sigma}_{n}=0.02(1-v)$. (b) Grain size $d=12000 b$, thickness $t=12000 b$ and $1500 b, \mathrm{HIGH}$ initial dislocation density case. $\tilde{\sigma}_{n}=0.02(1-v)$. (c) Grain size $d=3000 b$, thickness $t=12000 b$ and $3000 b$, LOW initial dislocation density case. $\tilde{\sigma}_{n}=0.04(1-v)$. 